\title{
Article
}

\section{Some Properties of the Arithmetic-Geometric Index}

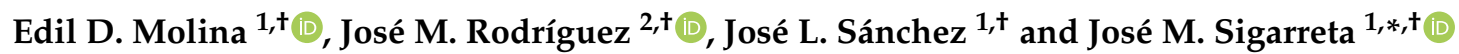 \\ 1 Facultad de Matemáticas, Universidad Autónoma de Guerrero, Carlos E. Adame No.54 Col. Garita, \\ 39650 Acalpulco Gro., Mexico; edi1941023@gmail.com (E.D.M.); jlsanchezsantiesteban@gmail.com (J.L.S.) \\ 2 Departamento de Matemáticas, Universidad Carlos III de Madrid, Avenida de la Universidad 30, \\ 28911 Leganés, Madrid, Spain; jomaro@math.uc3m.es \\ * Correspondence: josemariasigarretaalmira@hotmail.com; Tel.: +52-744-159-2272 \\ + These authors contributed equally to this work.
}

check for

updates

Citation: Molina, E.D.; Rodríguez, J.M.; Sánchez, J.L.; Sigarreta, J.M. Some Properties of the Arithmetic-Geometric Index. Symmetry 2021, 13, 857. https:// doi.org/10.3390/sym13050857

Academic Editor: Louis H. Kauffman

Received: 26 April 2021

Accepted: 10 May 2021

Published: 12 May 2021

Publisher's Note: MDPI stays neutral with regard to jurisdictional claims in published maps and institutional affiliations.

Copyright: (c) 2021 by the authors. Licensee MDPI, Basel, Switzerland. This article is an open access article distributed under the terms and conditions of the Creative Commons Attribution (CC BY) license (https:// creativecommons.org/licenses/by/ $4.0 /)$.

\begin{abstract}
Recently, the arithmetic-geometric index (AG) was introduced, inspired by the well-known and studied geometric-arithmetic index (GA). In this work, we obtain new bounds on the arithmeticgeometric index, improving upon some already known bounds. In particular, we show families of graphs where such bounds are attained.
\end{abstract}

Keywords: arithmetic-geometric index; topological index; chemical graph theory

\section{Introduction}

In mathematical chemistry, a topological descriptor is a function that associates each molecular graph with a real value, and if it correlates well with some chemical property, it is called a topological index. Since Wiener's work (see [1]), numerous topological indices have been defined and discussed, since the growing interest in their study is due to their several applications in chemistry, for example in QSPR/QSAR research (see [2-4]). For more information on other important applications of topological indices to specific problems in physics, computer science and environment science (see [5-7]). In particular, among the topological descriptors, the most studied from the mathematical point of view due to their practical scope are the so-called vertex-degree-based topological indices. Probably the most studied, with more than 500 papers, is the Randić index defined as

$$
R(H)=\sum_{i j \in E(H)} \frac{1}{\sqrt{d_{i} d_{j}}},
$$

where $i j$ denotes the edge of the graph $H$ and $d_{i}$ is the degree of the vertex $i$.

In $[8,9]$, the variable Zagreb indices are defined as

$$
M_{1}^{\alpha}(H)=\sum_{i \in V(H)} d_{i}^{\alpha}, \quad M_{2}^{\alpha}(H)=\sum_{i j \in E(H)}\left(d_{i} d_{j}\right)^{\alpha},
$$

with $\alpha \in \mathbb{R}$.

Note that for $\alpha=2, \alpha=-1, \alpha=3$, the index $M_{1}^{\alpha}$ is the first Zagreb index $M_{1}$, the inverse index $I D$, the forgotten index $F$, respectively; also for $\alpha=1, \alpha=-1 / 2, \alpha=-1$, the index $M_{2}^{\alpha}$ is the second Zagreb index $M_{2}$, the Randić index $R$, the modified Zagreb index.

The general sum-connectivity index was defined in [10] as

$$
\chi_{\alpha}(H)=\sum_{i j \in E(H)}\left(d_{i}+d_{j}\right)^{\alpha} .
$$

Note that $\chi_{-1 / 2}$ is the sum-connectivity index, $2 \chi_{-1}$ is the harmonic index Har, etc. The max-min rodeg index and min-max rodeg index were defined in [11] respectively as 


$$
M m_{\text {sde }}(H)=\sum_{i j \in E(H)} \sqrt{\frac{\max \left\{d_{i}, d_{j}\right\}}{\min \left\{d_{i}, d_{j}\right\}}}, \quad m M_{s d e}(H)=\sum_{i j \in E(H)} \sqrt{\frac{\min \left\{d_{i}, d_{j}\right\}}{\max \left\{d_{i}, d_{j}\right\}}} .
$$

these indices have shown good predictive properties (see [11]).

The symmetric division deg index was defined in $[11,12]$ as

$$
S D D(H)=\sum_{i j \in E(H)} \frac{d_{i}^{2}+d_{j}^{2}}{d_{i} d_{j}}=\sum_{i j \in E(H)}\left(\frac{d_{i}}{d_{j}}+\frac{d_{j}}{d_{i}}\right) .
$$

It was claimed in [11] that $S D D$ correlates well with the total surface area of polychlorobiphenyls. In the paper [13], the applicability of $S D D$ is tested on a wider empirical basis; also, its prediction ability is compared with other (more often used) topological indices.

The $G A(H)$ is defined in [14] as

$$
G A(H)=\sum_{i j \in E(H)} \frac{2 \sqrt{d_{i} d_{j}}}{d_{i}+d_{j}} .
$$

There are many papers studying the mathematical and computational properties of the GA index (see, e.g., [14-21] and the references therein).

As an inverse variant of this topological index, in 2015, the arithmetic-geometric index was introduced in [22] as

$$
A G(H)=\sum_{i j \in E(H)} \frac{d_{i}+d_{j}}{2 \sqrt{d_{i} d_{j}}} .
$$

The $A G$ index of some kinds of trees was discussed in the papers [22,23]. Moreover, the $A G$ index of graphene, which is the most conductive and effective material for electromagnetic interference shielding, was computed in [24]. The paper [25] studied the spectrum and energy of arithmetic-geometric matrix, in which the sum of all elements is equal to $2 A G$. Other bounds of the arithmetic-geometric energy of graphs appeared in $[26,27]$. The paper [28] studies optimal $A G$-graphs for several classes graphs, and it includes inequalities involving $G A+A G$ and $G A \cdot A G$. In [29-32], there are more bounds on the $A G$ index and a discussion on the effect of deleting an edge from a graph on the arithmetic-geometric index. Motivated by these papers, we obtain new bounds of the $A G$ index, improving upon some already known bounds. Furthermore, we show families of graphs where such bounds are attained. Some of these families are regular graphs, and we recall that some regular graphs play an important role in mathematical chemistry; for instance, Isaac graphs are well-known regular graphs that are isomorphic to hydrogen-suppressed molecular graphs [33].

Given a topological index $I(H)=\sum_{i j \in E(H)} f\left(d_{i}, d_{j}\right)$, we can consider the reciprocal topological index defined as $J(H)=\sum_{i j \in E(H)} 1 / f\left(d_{i}, d_{j}\right)$. It is essential to point out that several important topological indices are associated with the above relationships. For example, the first Zagreb index $M_{1}$ and the first modified Zagreb index ${ }^{m} M_{1}$, the second Zagreb index $M_{2}$ and the second modified Zagreb index ${ }^{m} M_{2}$, the Randić index $R$ and the reciprocal Randić index $M_{2}^{1 / 2}$, the max-min rodeg index $M m_{\text {sde }}$ and the min-max rodeg index $m M_{\text {sde }}$, etc.

Inspired by these ideas, the arithmetic-geometric index $A G$ was defined, which is the reciprocal of the well-studied geometric-arithmetic index $G A$. Although these topological indices are mathematically represented by an inverse relationship, their scope and results from both theoretical and practical points of view are different. In some cases, the reciprocal topological indices have shown better correlation with some physico-chemical properties than their related indices. In the case of the $A G$ index, in order to investigate its predictive power, we used a datum for entropy (S) of octane isomers, and the results are compared 
with those obtained for the $G A$ index, (see Figure 1). The correlation coefficient obtained for the $A G$ index is $\mathbf{r}_{A G}=-0.927$, while for the $G A$ index, it is $\mathbf{r}_{G A}=0.912$, so the $A G$ index, in this case, shows better predictive power than the $G A$ index. However, when we used a datum for the boiling point of octane isomers, it turned out that the $G A$ index showed better predictive power than the $A G$ index. After this paper was accepted, Ref. [34] showed that both indices have the same predictive power for many kinds of graphs.
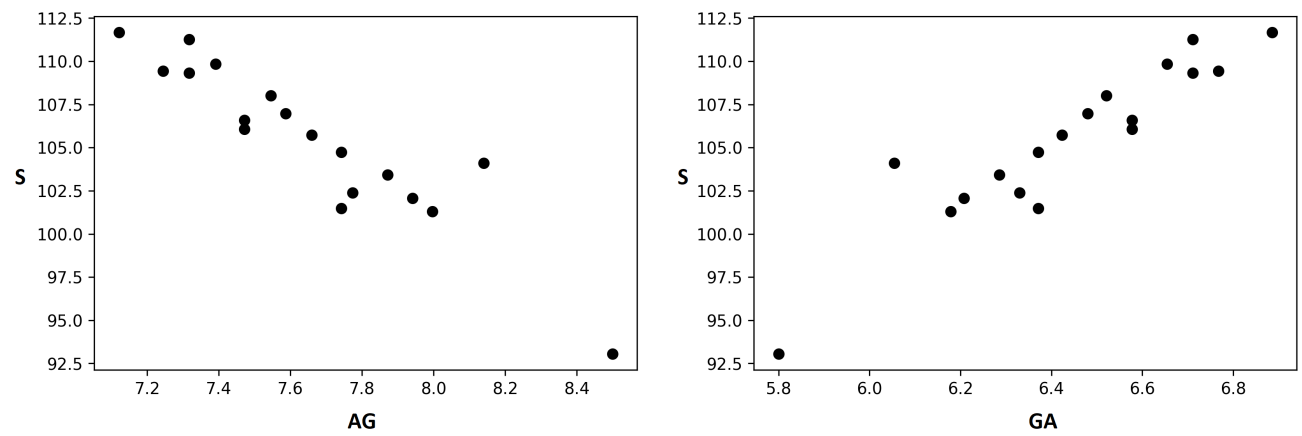

Figure 1. Graphs showing correlation between $\mathrm{S}$ and $A G, \mathrm{~S}$ and $G A$ respectively.

The arithmetic-geometric index was proposed recently and few important papers have been published on the subject. In this paper, we find several new mathematical properties (that cannot be obtained from the $G A$ index), especially bounds that improve those already known.

Throughout this work, $H=(V(H), E(H))$ denotes a finite simple graph with at least an edge in each connected component of $H$. We denote by $m, n, \delta, \Delta$ the cardinality of the set of edges $E(H)$ and vertices $V(H)$, and the minimum and maximum degree of $H$, respectively.

\section{Relationships between AG and Other Important Topological Indices}

One can check that the following lemma holds:

Lemma 1. Let $f$ be the function $f(x, y)=\frac{x+y}{2 \sqrt{x y}}$ defined on the rectangle $[a, b] \times[a, b]$ with $a>0$. Then:

$$
1 \leq f(x, y) \leq \frac{a+b}{2 \sqrt{a b}}
$$

The following inequalities for graphs $H$, follow from Lemma 1:

$$
m \leq A G(G) \leq \frac{\Delta+\delta}{2 \sqrt{\Delta \delta}} m
$$

The lower bound in (1) also follows from the inequalities $G A(H) \cdot A G(H) \geq m^{2}$ and $G A(H) \leq m$, see [15,16]. The upper bound in (1) appears in [31].

The following result shows the relationship between the $A G$ index and the Randic index that correlates well with several physico-chemical properties. For this reason, it is one of the most studied indices, with innumerable applications in chemistry and pharmacology.

Theorem 1. If $H$ is a graph with $m$ edges, minimum degree $\delta$ and maximum degree $\Delta$, then:

$$
A G(H) \leq m+\frac{(\sqrt{\Delta}-\sqrt{\delta})^{2}}{2} R(H) .
$$

The equality in the bound is attained if and only if $H$ is regular or biregular. 
Proof. Note that:

$$
\begin{aligned}
& \frac{d_{i}+d_{j}}{2 \sqrt{d_{i} d_{j}}}=1+\frac{\left(\sqrt{d_{i}}-\sqrt{d_{j}}\right)^{2}}{2 \sqrt{d_{i} d_{j}}}, \\
& A G(H)=m+\sum_{i j \in E(H)} \frac{\left(\sqrt{d_{i}}-\sqrt{d_{j}}\right)^{2}}{2 \sqrt{d_{i} d_{j}}} .
\end{aligned}
$$

Since:

$$
\sum_{i j \in E(H)} \frac{\left(\sqrt{d_{i}}-\sqrt{d_{j}}\right)^{2}}{2 \sqrt{d_{i} d_{j}}} \leq \frac{(\sqrt{\Delta}-\sqrt{\delta})^{2}}{2} \sum_{i j \in E(H)} \frac{1}{\sqrt{d_{i} d_{j}}}
$$

we have:

$$
A G(H) \leq m+\frac{(\sqrt{\Delta}-\sqrt{\delta})^{2}}{2} R(H) .
$$

The bound is tight if and only if:

$$
\left(\sqrt{d_{i}}-\sqrt{d_{j}}\right)^{2}=(\sqrt{\Delta}-\sqrt{\delta})^{2}
$$

for every $i j \in E(H)$, and this happens if and only if $d_{i}=\Delta$ and $d_{j}=\delta$, or vice versa, for every $i j \in E(H)$, so $H$ is regular if $\Delta=\delta$ or is otherwise biregular.

The following theorem shows a relationship between the index $A G$ and the index $M_{2}^{-a}$, the second variable Zagreb index.

Theorem 2. If $H$ is a graph with minimum degree $\delta$ and maximum degree $\Delta$, and $a \in \mathbb{R}$, then:

$$
A G(H) \leq K_{a} M_{2}^{-a}(H)
$$

with:

$$
K_{a}:= \begin{cases}\delta^{2 a}, & \text { if } a \leq-1 / 2, \\ \max \left\{\delta^{2 a}, \frac{1}{2}(\delta+\Delta)(\delta \Delta)^{a-1 / 2}\right\}, & \text { if }-1 / 2<a \leq 0, \\ \max \left\{\Delta^{2 a}, \frac{1}{2}(\delta+\Delta)(\delta \Delta)^{a-1 / 2}\right\}, & \text { if } 0<a<1 / 2, \\ \Delta^{2 a}, & \text { if } a \geq 1 / 2 .\end{cases}
$$

The equality in the bound is attained for some fixed $a \notin(-1 / 2,1 / 2)$ if and only if $H$ is a regular graph.

Proof. Let us optimize the function $g:[\delta, \Delta] \times[\delta, \Delta] \rightarrow(0, \infty)$ defined as

$$
g(x, y)=\frac{\frac{x+y}{2 \sqrt{x y}}}{(x y)^{-a}}=\frac{1}{2}(x y)^{a-1 / 2}(x+y)=\frac{1}{2} x^{a+1 / 2} y^{a-1 / 2}+\frac{1}{2} x^{a-1 / 2} y^{a+1 / 2}
$$

If $a \geq 1 / 2$, then $a+1 / 2>a-1 / 2 \geq 0$ and $g$ strictly increases in each variable. Thus:

$$
g(x, y) \leq g(\Delta, \Delta)=\Delta^{2 a}
$$

and the bound is tight if and only if $x=y=\Delta$. Therefore:

$$
A G(H) \leq \Delta^{2 a} M_{2}^{-a}(H) .
$$


Let us now consider the case $-1 / 2 \leq a<1 / 2$. Since $g$ is a symmetric function, we can also assume that $x \leq y$. We have:

$$
\begin{aligned}
\frac{\partial g}{\partial x}(x, y) & =\frac{1}{2}(1 / 2+a) x^{a-1 / 2} y^{a-1 / 2}+\frac{1}{2}(a-1 / 2) x^{a-3 / 2} y^{a+1 / 2} \\
& =\frac{1}{2} x^{a-3 / 2} y^{a-1 / 2}((1 / 2+a) x+(a-1 / 2) y), \\
\frac{\partial g}{\partial y}(x, y) & =\frac{1}{2} y^{a-3 / 2} x^{a-1 / 2}((1 / 2+a) y+(a-1 / 2) x) .
\end{aligned}
$$

Assume first that $0<a<1 / 2$. Thus, $a+1 / 2>0$ and:

$$
(1 / 2+a) y+(a-1 / 2) x \geq(1 / 2+a) x+(a-1 / 2) x=2 a x>0
$$

and thus, $\partial g / \partial y>0$. Therefore, the maximum value of $g$ is attained on $\{\delta \leq x \leq \Delta, y=\Delta\}$. Since:

$$
\frac{\partial g}{\partial x}(\Delta, \Delta)=\frac{1}{2} \Delta^{2 a-2}((a+1 / 2) \Delta+(a-1 / 2) \Delta)=a \Delta^{2 a-1}>0,
$$

and $\partial g / \partial x(x, \Delta)=0$ at most once when $x \in[\delta, \Delta]$, we have:

$$
\begin{aligned}
\max _{x, y \in[\delta, \Delta]} g(x, y) & =\max _{x \in[\delta, \Delta]} g(x, \Delta)=\max \{g(\delta, \Delta), g(\Delta, \Delta)\} \\
& =\max \left\{\frac{1}{2}(\Delta \delta)^{a-1 / 2}(\Delta+\delta), \Delta^{2 a}\right\} .
\end{aligned}
$$

Assume now that $-1 / 2<a \leq 0$. We have $a+1 / 2>0$ and:

$$
(1 / 2+a) x+(a-1 / 2) y \leq(1 / 2+a) y+(a-1 / 2) y=2 a y \leq 0
$$

and thus, $\partial g / \partial x \leq 0$. Therefore, the maximum value of $g$ is attained on $\{x=\delta, \delta \leq y \leq \Delta\}$. Since:

$$
\frac{\partial g}{\partial y}(\Delta, \Delta)=\frac{1}{2} \Delta^{2 a-2}((a+1 / 2) \Delta+(a-1 / 2) \Delta)=a \Delta^{2 a-1}>0,
$$

and $\partial g / \partial y(\delta, y)=0$ at most once when $y \in[\delta, \Delta]$, we have:

$$
\begin{aligned}
\max _{x, y \in[\delta, \Delta]} g(x, y) & =\max _{y \in[\delta, \Delta]} g(\delta, y)=\max \{g(\delta, \delta), g(\delta, \Delta)\} \\
& =\max \left\{\frac{1}{2}(\Delta \delta)^{a-1 / 2}(\Delta+\delta), \delta^{2 a}\right\} .
\end{aligned}
$$

Finally, assume that $a \leq-1 / 2$. Hence, $a-1 / 2<a+1 / 2 \leq 0$ and $g$ strictly decreases in each variable. Thus:

$$
g(x, y) \leq g(\delta, \delta)=\delta^{2 a}
$$

and the bound is tight if and only if $x=y=\delta$. Therefore:

$$
A G(H) \leq \delta^{2 a} M_{2}^{-a}(H) .
$$

The properties of the function $g$ give that the bound is tight for some fixed $a \geq 1 / 2$ (respectively, $a \leq-1 / 2$ ) if and only if $d_{i}=d_{j}=\Delta$ (respectively, $d_{i}=d_{j}=\delta$ ) for every $i j \in E(H)$, and this happens if and only if $H$ is a regular graph.

Remark 1. The proof of Theorem 2 allows us to obtain that:

$$
C_{a} M_{2}^{-a}(H) \leq A G(H)
$$


with:

$$
C_{a}:= \begin{cases}\Delta^{2 a}, & \text { if } a \leq 0, \\ \delta^{2 a}, & \text { if } a>0 .\end{cases}
$$

However, this inequality is direct, since:

$$
A G(H) \geq m=\sum_{i j \in E(H)} \frac{\left(d_{i} d_{j}\right)^{a}}{\left(d_{i} d_{j}\right)^{a}} \geq C_{a} \sum_{i j \in E(H)} \frac{1}{\left(d_{i} d_{j}\right)^{a}}=C_{a} M_{2}^{-a}(H) .
$$

Theorem 2 has the following result for the Randić, reciprocal Randić and modified Zagreb indices.

Corollary 1. If $H$ is a graph with a maximum degree $\Delta$ and minimum degree $\delta$, then:

$$
\begin{aligned}
& A G(H) \leq \delta^{-2} M_{2}(H), \\
& A G(H) \leq \Delta R(H), \\
& A G(H) \leq \delta^{-1} M_{2}^{1 / 2}(H), \\
& A G(H) \leq \Delta^{2} M_{2}^{-1}(H) .
\end{aligned}
$$

The following result shows a relationship between the $A G(H)$ index and the $\chi_{\beta}(H)$ index, which for different values of $b$ generalizes the indices $M_{1}$, Har, $\chi(b=1, b=-1$, $b=-1 / 2$, respectively).

Theorem 3. If $H$ is a graph with minimum degree $\delta$ and maximum degree $\Delta$, and $b \in \mathbb{R}$, then:

$$
A G(H) \leq B_{b} \chi_{b}(H)
$$

with:

$$
B_{b}:= \begin{cases}\max \left\{\frac{1}{2}(\Delta \delta)^{-1 / 2}(\Delta+\delta)^{1-b},(2 \Delta)^{-b}\right\}, & \text { if } b<0 \\ \max \left\{\frac{1}{2}(\Delta \delta)^{-1 / 2}(\Delta+\delta)^{1-b},(2 \delta)^{-b}\right\}, & \text { if } 0 \leq b<1 / 2 \\ (2 \delta)^{-b}, & \text { if } b \geq 1 / 2 .\end{cases}
$$

The equality in the bound is attained for some fixed $b \geq 1 / 2$ if and only if $H$ is a regular graph.

Proof. For each $b<1 / 2$, let us define:

$$
a=\frac{b}{2 b-2} \in\left(\frac{-1}{2}, \frac{1}{2}\right) .
$$

Let us consider the function: $g:[\delta, \Delta] \times[\delta, \Delta] \rightarrow(0, \infty)$ defined as

$$
g(x, y)=\frac{1}{2}(x y)^{a-1 / 2}(x+y)=\frac{1}{2} x^{a+1 / 2} y^{a-1 / 2}+\frac{1}{2} x^{a-1 / 2} y^{a+1 / 2} .
$$

Since $g$ is a symmetric function, we can assume $x \leq y$. We have:

$$
\begin{aligned}
\frac{\partial g}{\partial x}(x, y) & =\frac{1}{2}(1 / 2+a) x^{a-1 / 2} y^{a-1 / 2}+\frac{1}{2}(a-1 / 2) x^{a-3 / 2} y^{a+1 / 2} \\
& =\frac{1}{2} x^{a-3 / 2} y^{a-1 / 2}((1 / 2+a) x+(a-1 / 2) y), \\
\frac{\partial g}{\partial y}(x, y) & =\frac{1}{2} y^{a-3 / 2} x^{a-1 / 2}((1 / 2+a) y+(a-1 / 2) x) .
\end{aligned}
$$

Assume first that $0<a<1 / 2$. Thus, $1 / 2+a>0$ and:

$$
(1 / 2+a) y+(a-1 / 2) x \geq(1 / 2+a) x+(a-1 / 2) x=2 a x>0
$$


and thus, $\partial g / \partial y>0$. Therefore, the maximum value of $g$ is attained on $\{\delta \leq x \leq \Delta, y=\Delta\}$. Since:

$$
\frac{\partial g}{\partial x}(\Delta, \Delta)=\frac{1}{2} \Delta^{2 a-2}((a+1 / 2) \Delta+(a-1 / 2) \Delta)=a \Delta^{2 a-1}>0,
$$

and $\partial g / \partial x(x, \Delta)=0$ at most once when $x \in[\delta, \Delta]$, we have:

$$
\begin{aligned}
\max _{x, y \in[\delta, \Delta]} g(x, y) & =\max _{x \in[\delta, \Delta]} g(x, \Delta)=\max \{g(\delta, \Delta), g(\Delta, \Delta)\} \\
& =\max \left\{\frac{1}{2}(\Delta \delta)^{a-1 / 2}(\Delta+\delta), \Delta^{2 a}\right\} .
\end{aligned}
$$

Assume now that $-1 / 2<a \leq 0$. We have $a+1 / 2>0$ and:

$$
(1 / 2+a) x+(a-1 / 2) y \leq(1 / 2+a) y+(a-1 / 2) y=2 a y \leq 0
$$

and thus, $\partial g / \partial x \leq 0$. Therefore, the maximum value of $g$ is attained on $\{x=\delta, \delta \leq y \leq \Delta\}$. Since:

$$
\frac{\partial g}{\partial y}(\Delta, \Delta)=\frac{1}{2} \Delta^{2 a-2}((a+1 / 2) \Delta+(a-1 / 2) \Delta)=a \Delta^{2 a-1}>0,
$$

and $\partial g / \partial y(\delta, y)=0$ at most once when $y \in[\delta, \Delta]$, we have:

$$
\begin{aligned}
\max _{x, y \in[\delta, \Delta]} g(x, y) & =\max _{y \in[\delta, \Delta]} g(\delta, y)=\max \{g(\delta, \delta), g(\delta, \Delta)\} \\
& =\max \left\{\frac{1}{2}(\Delta \delta)^{a-1 / 2}(\Delta+\delta), \delta^{2 a}\right\} .
\end{aligned}
$$

Define:

$$
C_{a}:= \begin{cases}\max \left\{\delta^{2 a}, \frac{1}{2}(\delta+\Delta)(\delta \Delta)^{a-1 / 2}\right\}, & \text { if }-1 / 2<a \leq 0, \\ \max \left\{\Delta^{2 a}, \frac{1}{2}(\delta+\Delta)(\delta \Delta)^{a-1 / 2}\right\}, & \text { if } 0<a<1 / 2,\end{cases}
$$

we have:

$$
\begin{aligned}
(x y)^{a-1 / 2}(x+y) & \leq 2 C_{a}, \\
(x y)^{1 /(2 b-2)}(x+y) & \leq 2 C_{a} .
\end{aligned}
$$

Since $b<1 / 2$, we have $1-b>0$ and:

$$
\frac{1}{2}(x y)^{-1 / 2}(x+y)^{1-b} \leq \frac{1}{2}\left(2 C_{a}\right)^{1-b} .
$$

If $0 \leq b<1 / 2$, then $-1 / 2<a \leq 0$ and:

$$
\begin{aligned}
\frac{1}{2}\left(2 C_{a}\right)^{1-b} & =\frac{1}{2}\left(2 \max \left\{\frac{1}{2}(\Delta \delta)^{a-1 / 2}(\Delta+\delta), \delta^{2 a}\right\}\right)^{1-b} \\
& =\frac{1}{2}\left(2 \max \left\{\frac{1}{2}(\Delta \delta)^{1 /(2 b-2)}(\Delta+\delta), \delta^{b /(b-1)}\right\}\right)^{1-b} \\
& =\max \left\{\frac{1}{2}(\Delta \delta)^{-1 / 2}(\Delta+\delta)^{1-b},(2 \delta)^{-b}\right\}=B_{b} .
\end{aligned}
$$

If $b<0$, then $0<a<1 / 2$ and:

$$
\begin{aligned}
\frac{1}{2}\left(2 C_{a}\right)^{1-b} & =\frac{1}{2}\left(2 \max \left\{\frac{1}{2}(\Delta \delta)^{a-1 / 2}(\Delta+\delta), \Delta^{2 a}\right\}\right)^{1-b} \\
& =\frac{1}{2}\left(2 \max \left\{\frac{1}{2}(\Delta \delta)^{1 /(2 b-2)}(\Delta+\delta), \Delta^{b /(b-1)}\right\}\right)^{1-b} \\
& =\max \left\{\frac{1}{2}(\Delta \delta)^{-1 / 2}(\Delta+\delta)^{1-b},(2 \Delta)^{-b}\right\}=B_{b} .
\end{aligned}
$$


If $b \geq 1 / 2$, then the function $A:[\delta, \Delta] \times[\delta, \Delta] \rightarrow \mathbb{R}$ defined as

$$
A(x, y)=2 \sqrt{x y}(x+y)^{b-1}
$$

satisfies:

$$
\begin{aligned}
\frac{\partial A}{\partial x}(x, y) & =x^{-1 / 2} y^{1 / 2}(x+y)^{b-1}+2 x^{1 / 2} y^{1 / 2}(b-1)(x+y)^{b-2} \\
& =x^{-1 / 2} y^{1 / 2}(x+y)^{b-2}(x+y+(2 b-2) x)^{b-2} \\
& =x^{-1 / 2} y^{1 / 2}(x+y)^{b-2}((2 b-1) x+y)^{b-2} \\
& \geq x^{-1 / 2} y^{b-3 / 2}(x+y)^{b-2}>0, \\
\frac{\partial A}{\partial y}(x, y) & =y^{-1 / 2} x^{1 / 2}(x+y)^{b-2}((2 b-1) y+x)^{b-2} \\
& \geq y^{-1 / 2} x^{b-3 / 2}(x+y)^{b-2}>0 .
\end{aligned}
$$

Thus, $A$ is a strictly increasing function in each variable and thus:

$$
2 \sqrt{x y}(x+y)^{b-1}=A(x, y) \geq A(\delta, \delta)=(2 \delta)^{b},
$$

with equality if and only if $x=y=\delta$. Hence:

$$
\begin{aligned}
(2 \delta)^{b} \frac{x+y}{2 \sqrt{x y}} & \leq(x+y)^{b} \quad \forall x, y \in[\delta, \Delta], \\
\frac{d_{i}+d_{j}}{2 \sqrt{d_{i} d_{j}}} & \leq(2 \delta)^{-b}\left(d_{i}+d_{j}\right)^{b} \quad \forall i j \in E(H), \\
A G(H) & \leq B_{b} \chi_{b}(H),
\end{aligned}
$$

and the equality in this last inequality is attained if and only if $d_{i}=d_{j}=\delta$ for every $i j \in E(H)$, i.e., $H$ is a regular graph.

Remark 2. The proof of Theorem 3 allows us to obtain that:

$$
A_{b} \chi_{b}(H) \leq A G(H),
$$

with:

$$
A_{b}:= \begin{cases}(2 \delta)^{-b}, & \text { if } b<0, \\ (2 \Delta)^{-b}, & \text { if } b \geq 0 .\end{cases}
$$

However, this inequality is direct, since:

$$
A G(H) \geq m=\sum_{i j \in E(H)} \frac{\left(d_{i}+d_{j}\right)^{b}}{\left(d_{i}+d_{j}\right)^{b}} \geq A_{b} \sum_{i j \in E(H)}\left(d_{i}+d_{j}\right)^{b}=A_{b} \chi_{b}(H) .
$$

Theorem 3 has the following consequence for the first Zagreb, harmonic and sumconnectivity indices.

Corollary 2. Let $H$ be a graph with minimum degree $\delta$ and maximum degree $\Delta$. Then:

$$
\begin{aligned}
& A G(H) \leq \frac{1}{2 \delta} M_{1}(H), \\
& A G(H) \leq \frac{1}{2} \max \left\{\frac{1}{2}(\Delta \delta)^{-1 / 2}(\Delta+\delta)^{2}, 2 \Delta\right\} \operatorname{Har}(H), \\
& A G(H) \leq \max \left\{\frac{1}{2}(\Delta \delta)^{-1 / 2}(\Delta+\delta)^{3 / 2},(2 \Delta)^{1 / 2}\right\} \chi(H) .
\end{aligned}
$$

The following result relates $A G$ and $S D D$ indices. 
Theorem 4. Let $H$ be a graph with $m$ edges, minimum degree $\delta$ and maximum degree $\Delta$. Then:

$$
\frac{\sqrt{\Delta \delta}}{\Delta+\delta}\left(\frac{1}{2} S D D(H)+m\right) \leq A G(H) \leq \frac{1}{4} S D D(H)+\frac{1}{2} m .
$$

The equality in the lower bound is attained if $H$ is a regular or biregular graph. The equality in the upper bound is attained if and only if each connected component of $H$ is a regular graph.

Proof. Lemma 1 gives:

$$
\begin{aligned}
\frac{\Delta+\delta}{\sqrt{\Delta \delta}} A G(H) & =\sum_{i j \in E(H)} \frac{\Delta+\delta}{\sqrt{\Delta \delta}} \frac{d_{i}+d_{j}}{2 \sqrt{d_{i} d_{j}}} \geq \frac{1}{2} \sum_{i j \in E(H)}\left(\frac{d_{i}+d_{j}}{\sqrt{d_{i} d_{j}}}\right)^{2} \\
& =\frac{1}{2} \sum_{i j \in E(H)}\left(\sqrt{\frac{d_{i}}{d_{j}}}+\sqrt{\frac{d_{j}}{d_{i}}}\right)^{2}=\frac{1}{2} \sum_{i j \in E(H)}\left(\frac{d_{i}}{d_{j}}+\frac{d_{j}}{d_{i}}\right)+\frac{1}{2} \sum_{i j \in E(H)} 2 \\
& =\frac{1}{2} S D D(H)+m .
\end{aligned}
$$

If $H$ is a regular or biregular graph, then:

$$
\begin{aligned}
\frac{\sqrt{\Delta \delta}}{\Delta+\delta}\left(\frac{1}{2} S D D(H)+m\right) & =\frac{1}{2} \frac{\sqrt{\Delta \delta}}{\Delta+\delta}\left(\left(\frac{\Delta}{\delta}+\frac{\delta}{\Delta}\right) m+2 m\right) \\
& =\frac{1}{2} \frac{\sqrt{\Delta \delta}}{\Delta+\delta}\left(\frac{\Delta^{2}+\delta^{2}+2 \Delta \delta}{\Delta \delta}\right) m=\frac{\Delta+\delta}{2 \sqrt{\Delta \delta}} m=A G(H) .
\end{aligned}
$$

Lemma 1 gives:

$$
\begin{aligned}
A G(H) & =\sum_{i j \in E(H)} \frac{d_{i}+d_{j}}{2 \sqrt{d_{i} d_{j}}} \leq \frac{1}{4} \sum_{i j \in E(H)}\left(\frac{d_{i}+d_{j}}{\sqrt{d_{i} d_{j}}}\right)^{2} \\
& =\frac{1}{4} \sum_{i j \in E(H)}\left(\sqrt{\frac{d_{i}}{d_{j}}}+\sqrt{\frac{d_{j}}{d_{i}}}\right)^{2}=\frac{1}{4} \sum_{i j \in E(H)}\left(\frac{d_{i}}{d_{j}}+\frac{d_{j}}{d_{i}}\right)+\frac{1}{4} \sum_{i j \in E(H)} 2 \\
& =\frac{1}{4} S D D(H)+\frac{1}{2} m .
\end{aligned}
$$

If the equality in this bound is attained, then Lemma 1 gives $d_{i}=d_{j}$ for every $i j \in E(H)$ and so, each connected component of $H$ is a regular graph.

If each connected component of $H$ is a regular graph, then:

$$
\frac{1}{4} S D D(H)+\frac{1}{2} m=\frac{1}{4}(2 m+2 m)=m=A G(H) .
$$

It is easy to check that $\operatorname{SDD}(H) \geq 2 m$ and thus, Theorem 4 has the following consequence.

Corollary 3. Let $H$ be a graph. Then:

$$
A G(H) \leq \frac{1}{2} S D D(H) .
$$

The inequality in Corollary 3 appears in [30, Theorem 10] for connected graphs. (Note that the definition of SDD in [30] is slightly different.) Our argument gives it for general graphs, and Theorem 4 improves this inequality. 
We present here elementary relations between $A G, M m_{\text {sde }}$ and $M m_{\text {sde }}$ indices.

Proposition 1. If $H$ is a graph, then:

$$
A G(H)=\frac{1}{2} M m_{s d e}(H)+\frac{1}{2} m M_{s d e}(H), \quad A G(H) \leq M m_{s d e}(H) .
$$

The equality in the bound is attained if and only if each connected component of $H$ is a regular graph.

Proof. We have:

$$
\begin{aligned}
A G(H) & =\sum_{i j \in E(H)} \frac{d_{i}+d_{j}}{2 \sqrt{d_{i} d_{j}}}=\frac{1}{2} \sum_{i j \in E(H)}\left(\sqrt{\frac{d_{i}}{d_{j}}}+\sqrt{\frac{d_{j}}{d_{i}}}\right) \\
& =\frac{1}{2} \sum_{i j \in E(H)}\left(\sqrt{\frac{\max \left\{d_{i}, d_{j}\right\}}{\min \left\{d_{i}, d_{j}\right\}}}+\sqrt{\frac{\min \left\{d_{i}, d_{j}\right\}}{\max \left\{d_{i}, d_{j}\right\}}}\right) \\
& =\frac{1}{2} M m_{s d e}(H)+\frac{1}{2} m M_{\text {sde }}(H) .
\end{aligned}
$$

In addition:

$$
A G(H)=\sum_{i j \in E(H)} \frac{1}{2}\left(\sqrt{\frac{d_{i}}{d_{j}}}+\sqrt{\frac{d_{j}}{d_{i}}}\right) \leq \sum_{i j \in E(H)} \sqrt{\frac{\max \left\{d_{i}, d_{j}\right\}}{\min \left\{d_{i}, d_{j}\right\}}}=M m_{s d e}(H)
$$

The bound is tight if and only if:

$$
\sqrt{\frac{d_{i}}{d_{j}}}=\sqrt{\frac{d_{j}}{d_{i}}}=\sqrt{\frac{\max \left\{d_{i}, d_{j}\right\}}{\min \left\{d_{i}, d_{j}\right\}}}
$$

for every $i j \in E(H)$, i.e., $d_{i}=d_{j}$ for every $i j \in E(H)$, and this happens if and only if each connected component of $H$ is a regular graph.

\section{A General Bound of the $A G$ Index}

In this section. we find and show optimal inequalities, which do not involve other topological indices, for the topological index $A G$ as a function of graph invariants such as the number of edges and the minimum and maximum degree.

We will need the following definitions. Given a graph $H$ with maximum degree $\Delta$ and minimum degree $\delta<\Delta-1$, we denote by $\alpha_{0}, \alpha_{1}, \alpha_{2}$, the cardinality of the subsets of edges

$$
\begin{aligned}
& A_{0}=\left\{i j \in E(H): d_{i}=\delta, d_{j}=\Delta\right\}, \\
& A_{1}=\left\{i j \in E(H): d_{i}=\delta, \delta<d_{i}<\Delta\right\}, \\
& A_{2}=\left\{i j \in E(H): d_{i}=\Delta, \delta<d_{j}<\Delta\right\},
\end{aligned}
$$

respectively.

Theorem 5. Let $H$ be a graph with maximum degree $\Delta$, minimum degree $\delta<\Delta-1$ and m edges. Then:

$$
\begin{aligned}
& A G(H) \leq \frac{\Delta+\delta}{2 \sqrt{\Delta \delta}} m-\alpha_{1}\left(\frac{\Delta+\delta}{2 \sqrt{\Delta \delta}}-\frac{\Delta+\delta-1}{2 \sqrt{(\Delta-1) \delta}}\right)-\alpha_{2}\left(\frac{\Delta+\delta}{2 \sqrt{\Delta \delta}}-\frac{\Delta+\delta+1}{2 \sqrt{\Delta(\delta+1)}}\right) \\
& A G(H) \geq m+\alpha_{0}\left(\frac{\delta+\Delta}{2 \sqrt{\delta \Delta}}-1\right)+\alpha_{1}\left(\frac{1+2 \delta}{2 \sqrt{(\delta+1) \delta}}-1\right)+\alpha_{2}\left(\frac{2 \Delta-1}{2 \sqrt{(\Delta-1) \Delta}}-1\right) .
\end{aligned}
$$


Proof. Let us consider the function $g(t)=\frac{1+t^{2}}{2 t}$ on the interval $(0, \infty)$. We have $g^{\prime}(t)=\frac{t^{2}-1}{2 t^{2}}$, therefore $g^{\prime}(t)<0$ for $t \in(0,1)$ and $g^{\prime}(t)>0$ for $t \in(1, \infty)$. Then, $g$ decreases on $(0,1]$ and $g$ increases on $[1, \infty)$.

From the above argument, it follows that the function:

$$
\frac{\delta+d_{j}}{2 \sqrt{\delta d_{j}}}=g\left(\left(\frac{d_{j}}{\delta}\right)^{1 / 2}\right)
$$

is increasing in $d_{j} \in(\delta, \Delta)$ and thus:

$$
\frac{\delta+(\delta+1)}{2 \sqrt{\delta(\delta+1)}} \leq \frac{\delta+d_{j}}{2 \sqrt{\delta d_{j}}} \leq \frac{\delta+\Delta-1}{2 \sqrt{\delta(\Delta-1)}},
$$

for every $i j \in A_{1}$.

In a similar way, the function:

$$
\frac{\Delta+d_{j}}{2 \sqrt{\Delta d_{j}}}=g\left(\left(\frac{d_{j}}{\Delta}\right)^{1 / 2}\right)
$$

is decreasing in $d_{j} \in(\delta, \Delta)$ and thus:

$$
\frac{\Delta+(\Delta-1)}{2 \sqrt{\Delta(\Delta-1)}} \leq \frac{\Delta+d_{j}}{2 \sqrt{\Delta d_{j}}} \leq \frac{\Delta+\delta+1}{2 \sqrt{\Delta(\delta+1)}}
$$

for every $i j \in A_{2}$.

Since:

$$
1 \leq \frac{d_{i}+d_{j}}{2 \sqrt{d_{i} d_{j}}} \leq \frac{\Delta+\delta}{2 \sqrt{\Delta \delta}}
$$

for every $i j \in E(H)$, we have:

$$
\begin{aligned}
A G(H) & =\sum_{i j \in E(H) \backslash A_{0} \cup A_{1} \cup A_{2}} \frac{d_{i}+d_{j}}{2 \sqrt{d_{i} d_{j}}}+\sum_{i j \in A_{0}} \frac{d_{i}+d_{j}}{2 \sqrt{d_{i} d_{j}}}+\sum_{i j \in A_{1}} \frac{d_{i}+d_{j}}{2 \sqrt{d_{i} d_{j}}}+\sum_{i j \in A_{2}} \frac{d_{i}+d_{j}}{2 \sqrt{d_{i} d_{j}}} \\
& =\sum_{i j \in E(H) \backslash A_{0} \cup A_{1} \cup A_{2}} \frac{d_{i}+d_{j}}{2 \sqrt{d_{i} d_{j}}}+\sum_{i j \in A_{0}} \frac{\Delta+\delta}{2 \sqrt{\Delta \delta}}+\sum_{i j \in A_{1}} \frac{\delta+d_{j}}{2 \sqrt{\delta d_{j}}}+\sum_{i j \in A_{2}} \frac{\Delta+d_{j}}{2 \sqrt{\Delta d_{j}}}
\end{aligned}
$$

therefore:

$$
A G(H) \geq m-\alpha_{0}-\alpha_{1}-\alpha_{2}+\alpha_{0} \frac{\Delta+\delta}{2 \sqrt{\Delta \delta}}+\alpha_{1} \frac{2 \delta+1}{2 \sqrt{\delta(\delta+1)}}+\alpha_{2} \frac{2 \Delta-1}{2 \sqrt{\Delta(\Delta-1)}},
$$

and:

$$
\begin{aligned}
A G(H) & \leq\left(m-\alpha_{0}-\alpha_{1}-\alpha_{2}\right) \frac{\Delta+\delta}{2 \sqrt{\Delta \delta}}+\alpha_{0} \frac{\Delta+\delta}{2 \sqrt{\Delta \delta}}+\alpha_{1} \frac{\Delta+\delta-1}{2 \sqrt{(\Delta-1) \delta}}+\alpha_{2} \frac{\Delta+\delta+1}{2 \sqrt{\Delta(\delta+1)}} \\
& =\frac{\Delta+\delta}{2 \sqrt{\Delta \delta}} m-\alpha_{1}\left(\frac{\Delta+\delta}{2 \sqrt{\Delta \delta}}-\frac{\Delta+\delta-1}{2 \sqrt{\delta(\Delta-1)}}\right)-\alpha_{2}\left(\frac{\Delta+\delta}{2 \sqrt{\Delta \delta}}-\frac{\delta+\Delta+1}{2 \sqrt{\Delta(\delta+1)}}\right) .
\end{aligned}
$$

Lemma 2. If $v(t)=\frac{1+t}{2 \sqrt{t}}$, then: $(1) v(t) \leq \frac{1}{8}(1-t)^{2}+1$ for every $t \in[1, \infty)$, (2) $v(t) \geq \frac{1}{16}(1-t)^{2}+1$ for every $t \in(0,1.945]$. 
Proof. We have for every $s \in[1, \infty)$ and $t=s^{2} \in[1, \infty)$ :

$$
\begin{aligned}
(s-1)^{3}\left(s^{2}+3 s+4\right) & \geq 0, \\
s^{5}-2 s^{3}-4 s^{2}+9 s-4 & \geq 0, \\
4\left(s^{2}+1\right) & \leq s\left(8+\left(1-s^{2}\right)^{2}\right), \\
v(t)=\frac{t+1}{2 \sqrt{t}} & \leq \frac{1}{8}(1-t)^{2}+1 .
\end{aligned}
$$

Let $s_{1}=1.39485 \ldots$ be the unique real solution of $s^{3}+2 s^{2}+s-8=0$ in the interval $(0, \infty)$. We have for every $s \in\left[0, s_{1}\right]$ and $t=s^{2} \in(0,1.945] \subset\left(0, s_{1}^{2}\right]$ :

$$
\begin{aligned}
(s-1)^{2}\left(s^{3}+2 s^{2}+s-8\right) & \leq 0 \\
s^{5}-2 s^{3}-8 s^{2}+17 s-8 & \leq 0 \\
8\left(s^{2}+1\right) & \geq s\left(16+\left(1-s^{2}\right)^{2}\right), \\
v(t)=\frac{1+t}{2 \sqrt{t}} & \geq \frac{1}{16}(1-t)^{2}+1 .
\end{aligned}
$$

Proposition 2. Let $H$ be a graph with maximum degree $\Delta$, minimum degree $\delta<\Delta-1$ and medges.

1. If $\delta$ is an even integer, then:

$$
A G(H) \geq m+\min \left\{\frac{\Delta+\delta}{2 \sqrt{\Delta \delta}}+\frac{2 \delta+1}{2 \sqrt{\delta(\delta+1)}}-2, \frac{2 \delta+1}{\sqrt{\delta(\delta+1)}}+\frac{2 \Delta-1}{2 \sqrt{\Delta(\Delta-1)}}-3\right\} .
$$

2. If $\Delta$ is an even integer, then:

$$
A G(H) \geq m+\min \left\{\frac{\Delta+\delta}{2 \sqrt{\Delta \delta}}+\frac{2 \Delta-1}{2 \sqrt{\Delta(\Delta-1)}}-2, \frac{2 \delta+1}{2 \sqrt{\delta(\delta+1)}}+\frac{2 \Delta-1}{\sqrt{\Delta(\Delta-1)}}-3\right\} .
$$

3. If $\delta$ and $\Delta$ are even integers, then:

$$
A G(H) \geq m+\frac{2 \delta+1}{\sqrt{\delta(\delta+1)}}+\frac{2 \Delta-1}{\sqrt{\Delta(\Delta-1)}}-4 .
$$

Proof. Assume first that $\delta$ is an even integer.

Let $H_{1}$ be the subgraph of $H$ induced by the $n_{1}$ vertices with degree $\delta$ in $V(H)$, and denote by $m_{1}$ the cardinality of the set of edges of $H_{1}$. Handshaking Lemma gives $n_{1} \delta-\alpha_{0}-\alpha_{1}=2 m_{1}$. Since $\delta$ is an even integer, $\alpha_{0}+\alpha_{1}$ is also an even integer; since each component of $H$ is a connected graph, we have $\alpha_{0}+\alpha_{1} \geq 1$ and so, $\alpha_{0}+\alpha_{1} \geq 2$.

If $\alpha_{0} \geq 2$, then Theorem 5 gives:

$$
\begin{aligned}
A G(H) & \geq m+\alpha_{0}\left(\frac{\delta+\Delta}{2 \sqrt{\delta \Delta}}-1\right)+\alpha_{1}\left(\frac{1+2 \delta}{2 \sqrt{(\delta+1) \delta}}-1\right)+\alpha_{2}\left(\frac{2 \Delta-1}{2 \sqrt{(\Delta-1) \Delta}}-1\right) \\
& \geq m+\frac{\delta+\Delta}{\sqrt{\delta \Delta}}-2 .
\end{aligned}
$$


If $\alpha_{0}=1$, then $\alpha_{1} \geq 1$ and Theorem 5 gives:

$$
\begin{aligned}
A G(H) & \geq m+\alpha_{0}\left(\frac{\delta+\Delta}{2 \sqrt{\delta \Delta}}-1\right)+\alpha_{1}\left(\frac{1+2 \delta}{2 \sqrt{(\delta+1) \delta}}-1\right)+\alpha_{2}\left(\frac{2 \Delta-1}{2 \sqrt{(\Delta-1) \Delta}}-1\right) \\
& \geq m+\frac{\Delta+\delta}{2 \sqrt{\Delta \delta}}+\frac{2 \delta+1}{2 \sqrt{\delta(\delta+1)}}-2 .
\end{aligned}
$$

If $\alpha_{0}=0$, then $\alpha_{1} \geq 2$ and $\alpha_{2} \geq 1$, and Theorem 5 gives:

$$
\begin{aligned}
A G(H) & \geq m+\alpha_{0}\left(\frac{\delta+\Delta}{2 \sqrt{\delta \Delta}}-1\right)+\alpha_{1}\left(\frac{1+2 \delta}{2 \sqrt{(\delta+1) \delta}}-1\right)+\alpha_{2}\left(\frac{2 \Delta-1}{2 \sqrt{(\Delta-1) \Delta}}-1\right) \\
& \geq m+\frac{2 \delta+1}{\sqrt{\delta(\delta+1)}}+\frac{2 \Delta-1}{2 \sqrt{\Delta(\Delta-1)}}-3 .
\end{aligned}
$$

Since Lemma 1 gives:

$$
\frac{\Delta+\delta}{2 \sqrt{\Delta \delta}} \geq \frac{2 \delta+1}{2 \sqrt{\delta(\delta+1)}}
$$

we have:

$$
A G(H) \geq m+\min \left\{\frac{\delta+\Delta}{2 \sqrt{\delta \Delta}}+\frac{2 \delta+1}{2 \sqrt{(\delta+1) \delta}}-2, \frac{2 \delta+1}{\sqrt{(\delta+1) \delta}}+\frac{2 \Delta-1}{2 \sqrt{\Delta(\Delta-1)}}-3\right\} .
$$

Assume now that $\Delta$ is an even integer. Let $H_{2}$ be the subgraph of $H$ induced by the $n_{2}$ vertices with a degree $\Delta$ in $V(H)$, and denote by $m_{2}$ the cardinality of the set of edges of $H_{2}$. Handshaking Lemma gives $n_{2} \Delta-\alpha_{0}-\alpha_{2}=2 m_{2}$. Since $\Delta$ is an even integer, $\alpha_{0}+\alpha_{2}$ is also an even integer; since each component of $H$ is a connected graph, we have $\alpha_{0}+\alpha_{2} \geq 1$ and thus, $\alpha_{0}+\alpha_{2} \geq 2$.

If $\alpha_{0} \geq 2$, then Theorem 5 gives:

$$
\begin{aligned}
A G(H) & \geq m+\alpha_{0}\left(\frac{\delta+\Delta}{2 \sqrt{\delta \Delta}}-1\right)+\alpha_{1}\left(\frac{1+2 \delta}{2 \sqrt{(\delta+1) \delta}}-1\right)+\alpha_{2}\left(\frac{2 \Delta-1}{2 \sqrt{(\Delta-1) \Delta}}-1\right) \\
& \geq m+\frac{\Delta+\delta}{\sqrt{\Delta \delta}}-2 .
\end{aligned}
$$

If $\alpha_{0}=1$, then $\alpha_{2} \geq 1$ and Theorem 5 gives:

$$
\begin{aligned}
A G(H) & \geq m+\alpha_{0}\left(\frac{\delta+\Delta}{2 \sqrt{\delta \Delta}}-1\right)+\alpha_{1}\left(\frac{1+2 \delta}{2 \sqrt{(\delta+1) \delta}}-1\right)+\alpha_{2}\left(\frac{2 \Delta-1}{2 \sqrt{(\Delta-1) \Delta}}-1\right) \\
& \geq m+\frac{\Delta+\delta}{2 \sqrt{\Delta \delta}}+\frac{2 \Delta-1}{2 \sqrt{\Delta(\Delta-1)}}-2 .
\end{aligned}
$$

If $\alpha_{0}=0$, then $\alpha_{2} \geq 2$ and $\alpha_{1} \geq 1$, and Theorem 5 gives:

$$
\begin{aligned}
A G(H) & \geq m+\alpha_{0}\left(\frac{\delta+\Delta}{2 \sqrt{\delta \Delta}}-1\right)+\alpha_{1}\left(\frac{1+2 \delta}{2 \sqrt{(\delta+1) \delta}}-1\right)+\alpha_{2}\left(\frac{2 \Delta-1}{2 \sqrt{(\Delta-1) \Delta}}-1\right) \\
& \geq m+\frac{1+2 \delta}{2 \sqrt{(\delta+1) \delta}}+\frac{2 \Delta-1}{\sqrt{\Delta(\Delta-1)}}-3 .
\end{aligned}
$$

Since:

$$
\frac{\Delta+\delta}{2 \sqrt{\Delta \delta}} \geq \frac{2 \Delta-1}{2 \sqrt{\Delta(\Delta-1)}}
$$


we have:

$$
A G(H) \geq m+\min \left\{\frac{\Delta+\delta}{2 \sqrt{\Delta \delta}}+\frac{2 \Delta-1}{2 \sqrt{\Delta(\Delta-1)}}-2, \frac{2 \delta+1}{2 \sqrt{\delta(\delta+1)}}+\frac{2 \Delta-1}{\sqrt{\Delta(\Delta-1)}}-3\right\} .
$$

Finally, assume that $\delta$ and $\Delta$ are even integers. The previous arguments give $\alpha_{0}+\alpha_{1} \geq 2$ and $\alpha_{0}+\alpha_{2} \geq 2$.

If $\alpha_{0} \geq 2$, then Theorem 5 gives:

$$
\begin{aligned}
A G(H) & \geq m+\alpha_{0}\left(\frac{\delta+\Delta}{2 \sqrt{\delta \Delta}}-1\right)+\alpha_{1}\left(\frac{1+2 \delta}{2 \sqrt{(\delta+1) \delta}}-1\right)+\alpha_{2}\left(\frac{2 \Delta-1}{2 \sqrt{(\Delta-1) \Delta}}-1\right) \\
& \geq m+\frac{\Delta+\delta}{\sqrt{\Delta \delta}}-2 .
\end{aligned}
$$

If $\alpha_{0}=1$, then $\alpha_{1}, \alpha_{2} \geq 1$ and Theorem 5 gives:

$$
\begin{aligned}
A G(H) & \geq m+\alpha_{0}\left(\frac{\delta+\Delta}{2 \sqrt{\delta \Delta}}-1\right)+\alpha_{1}\left(\frac{1+2 \delta}{2 \sqrt{(\delta+1) \delta}}-1\right)+\alpha_{2}\left(\frac{2 \Delta-1}{2 \sqrt{(\Delta-1) \Delta}}-1\right) \\
& \geq m+\frac{\Delta+\delta}{2 \sqrt{\Delta \delta}}+\frac{2 \delta+1}{2 \sqrt{\delta(\delta+1)}}+\frac{2 \Delta-1}{2 \sqrt{\Delta(\Delta-1)}}-3 .
\end{aligned}
$$

If $\alpha_{0}=0$, then $\alpha_{1}, \alpha_{2} \geq 2$, and Theorem 5 gives:

$$
\begin{aligned}
A G(H) & \geq m+\alpha_{0}\left(\frac{\Delta+\delta}{2 \sqrt{\Delta \delta}}-1\right)+\alpha_{1}\left(\frac{2 \delta+1}{2 \sqrt{\delta(\delta+1)}}-1\right)+\alpha_{2}\left(\frac{2 \Delta-1}{2 \sqrt{\Delta(\Delta-1)}}-1\right) \\
& \geq m+\frac{2 \delta+1}{\sqrt{\delta(\delta+1)}}+\frac{2 \Delta-1}{\sqrt{\Delta(\Delta-1)}}-4 .
\end{aligned}
$$

We claim now:

$$
1+\frac{\delta+\Delta}{2 \sqrt{\delta \Delta}} \geq \frac{1+2 \delta}{2 \sqrt{(\delta+1) \delta}}+\frac{2 \Delta-1}{2 \sqrt{(\Delta-1) \Delta}} .
$$

Assuming that this inequality holds, we have:

$$
\begin{gathered}
m+\frac{\delta+\Delta}{\sqrt{\delta \Delta}}-2 \geq m+\frac{\delta+\Delta}{2 \sqrt{\delta \Delta}}+\frac{1+2 \delta}{2 \sqrt{(\delta+1) \delta}}+\frac{2 \Delta-1}{2 \sqrt{(\Delta-1) \Delta}}-3, \\
m+\frac{\Delta+\delta}{2 \sqrt{\Delta \delta}}+\frac{2 \delta+1}{2 \sqrt{(\delta+1) \delta}}+\frac{2 \Delta-1}{2 \sqrt{(\Delta-1) \Delta}}-3 \geq m+\frac{1+2 \delta}{\sqrt{(\delta+1) \delta}}+\frac{2 \Delta-1}{\sqrt{(\Delta-1) \Delta}}-4,
\end{gathered}
$$

and we conclude:

$$
A G(H) \geq m+\frac{1+2 \delta}{\sqrt{(\delta+1) \delta}}+\frac{2 \Delta-1}{\sqrt{\Delta(\Delta-1)}}-4 .
$$

Thus, it suffices to prove the claim.

$$
\begin{aligned}
& 1+\frac{\delta+\Delta}{2 \sqrt{\delta \Delta}} \geq \frac{2 \delta+1}{2 \sqrt{\delta(\delta+1)}}+\frac{2 \Delta-1}{2 \sqrt{\Delta(\Delta-1)}}, \\
& 1+v\left(\frac{\Delta}{\delta}\right) \geq v\left(\frac{1+\delta}{\delta}\right)+v\left(\frac{\Delta}{\Delta-1}\right),
\end{aligned}
$$


where $v(t)=\frac{1+t}{2 \sqrt{t}}$ is the function in Lemma 2. Since $v$ is an increasing function in $[1, \infty)$ and $\Delta \geq \delta+2$, we have:

$$
v\left(\frac{2+\delta}{\delta}\right) \leq v\left(\frac{\Delta}{\delta}\right), \quad v\left(\frac{\Delta}{\Delta-1}\right) \leq v\left(\frac{2+\delta}{1+\delta}\right) .
$$

Hence, it suffices to show:

$$
1+v\left(\frac{2+\delta}{\delta}\right) \geq v\left(\frac{1+\delta}{\delta}\right)+v\left(\frac{2+\delta}{1+\delta}\right),
$$

for every $\delta \geq 1$.

Note that (2) holds for $\delta=1,2$. Let us prove that it holds for $\delta \geq 3$. Note that:

$$
\frac{1 / 8}{(\delta+1)^{2}} \leq \frac{1 / 8}{\delta^{2}}, \quad 2+\frac{1 / 8}{\delta^{2}}+\frac{1 / 8}{(\delta+1)^{2}} \leq 2+\frac{1 / 4}{\delta^{2}} .
$$

Since $\delta \geq 3$, we have:

$$
\frac{2+\delta}{\delta} \leq \frac{5}{3}<1.945, \quad \frac{1+\delta}{\delta}>1, \quad \frac{2+\delta}{1+\delta}>1 .
$$

Thus, Lemma 2 gives:

$$
\begin{aligned}
v\left(\frac{1+\delta}{\delta}\right)+v\left(\frac{2+\delta}{1+\delta}\right) & \leq 1+\frac{1}{8}\left(\frac{1+\delta}{\delta}-1\right)^{2}+1+\frac{1}{8}\left(\frac{2+\delta}{1+\delta}-1\right)^{2} \\
& =2+\frac{1 / 8}{\delta^{2}}+\frac{1 / 8}{(\delta+1)^{2}} \leq 2+\frac{1 / 4}{\delta^{2}} \\
& =1+1+\frac{1}{16}\left(1-\frac{2+\delta}{\delta}\right)^{2} \leq 1+v\left(\frac{2+\delta}{\delta}\right) .
\end{aligned}
$$

These inequalities give (2) for $\delta \geq 3$, and the proof is finished.

Finally, we show that the bound in Proposition 2 (3) is tight: let us consider the complete graphs $K_{5}$ and $K_{3}$, and fix $u_{1}, u_{2} \in V\left(K_{5}\right)$ and $v_{1}, v_{2} \in V\left(K_{3}\right)$. Denote by $K_{5}^{*}$ the graph obtained from $K_{5}$ by deleting the edge $u_{1} u_{2}$. Let $\Gamma$ be the graph with $V(\Gamma)=V\left(K_{5}^{*}\right) \cup$ $V\left(K_{3}\right)$ and $E(\Gamma)=E\left(K_{5}^{*}\right) \cup E\left(K_{3}\right) \cup\left\{u_{1} v_{1}\right\} \cup\left\{u_{2} v_{2}\right\}$. Thus, $\Gamma$ has a maximum degree $\Delta=4$, minimum degree $\delta=2, \alpha_{0}=0, \alpha_{1}=2, \alpha_{2}=2$; in addition, if $i j \notin A_{0} \cup A_{1} \cup A_{2}$, then $d_{i}=d_{j}$, if $i j \in A_{1}$, then $\left\{d_{i}, d_{j}\right\}=\{\delta, \delta+1\}$, and if $i j \in A_{2}$, then $\left\{d_{i}, d_{j}\right\}=\{\Delta, \Delta-1\}$. Then, we have:

$$
A G(\Gamma)=m+\frac{2 \delta+1}{\sqrt{\delta(\delta+1)}}+\frac{2 \Delta-1}{\sqrt{\Delta(\Delta-1)}}-4 .
$$

\section{Conclusions}

Topological indices have become a useful tool for the study of theoretical and practical problems in different areas of science. An important line of research associated with topological indices is that of determining optimal bounds and relations between known topological indices-particularly to obtain bounds for the topological indices associated with the invariant parameters of a graph.

Ref. [35] proves that many upper bounds of $G A$ are not useful, and shows the importance of obtaining upper bounds of $G A$ that are less than $m$. In a similar way, it is important to find lower bounds of $A G$ greater than $m$.

With this aim, we obtain in this paper several new lower bounds of $A G$, which are greater than $m$ for graphs with a maximum degree $\Delta$ and minimum degree $\delta<\Delta-1$ : 
1. If $\delta$ is an even integer, then:

$$
A G(H) \geq m+\min \left\{\frac{\Delta+\delta}{2 \sqrt{\Delta \delta}}+\frac{2 \delta+1}{2 \sqrt{\delta(\delta+1)}}-2, \frac{2 \delta+1}{\sqrt{\delta(\delta+1)}}+\frac{2 \Delta-1}{2 \sqrt{\Delta(\Delta-1)}}-3\right\} .
$$

2. If $\Delta$ is an even integer, then:

$$
A G(H) \geq m+\min \left\{\frac{\Delta+\delta}{2 \sqrt{\Delta \delta}}+\frac{2 \Delta-1}{2 \sqrt{\Delta(\Delta-1)}}-2, \frac{2 \delta+1}{2 \sqrt{\delta(\delta+1)}}+\frac{2 \Delta-1}{\sqrt{\Delta(\Delta-1)}}-3\right\} .
$$

3. If $\delta$ and $\Delta$ are even integers, then:

$$
A G(H) \geq m+\frac{2 \delta+1}{\sqrt{\delta(\delta+1)}}+\frac{2 \Delta-1}{\sqrt{\Delta(\Delta-1)}}-4 .
$$

We obtain several inequalities relating $A G$ with other topological indices, as

$$
\frac{\sqrt{\Delta \delta}}{\Delta+\delta}\left(\frac{1}{2} S D D(H)+m\right) \leq A G(H) \leq \frac{1}{4} S D D(H)+\frac{1}{2} m
$$

This result improves the following bound already known in the literature:

$$
A G(H) \leq \frac{1}{2} S D D(H)
$$

Moreover, we find families of graphs where the bounds are attained.

Furthermore, we show that at least for entropy, the $A G$ index has better predictive power than $G A$, while for other physicochemical properties, the $G A$ index has better predictive power than $A G$.

We think that it would be interesting to obtain for the geometric-arithmetic index some results similar to those included in this work for the $A G$ index.

Author Contributions: Investigation, E.D.M., J.M.R., J.L.S. and J.M.S. All authors have read and agreed to the published version of the manuscript.

Funding: This research was supported by a grant from Agencia Estatal de Investigación (PID2019106433GBI00/ AEI/10.13039/501100011033), Spain. The research of José M. Rodríguez was supported by the Madrid Government (Comunidad de Madrid-Spain) under the Multiannual Agreement with UC3M in the line of Excellence of University Professors (EPUC3M23), and in the context of the V PRICIT (Regional Programme of Research and Technological Innovation).

Institutional Review Board Statement: Not applicable.

Informed Consent Statement: Not applicable.

Data Availability Statement: Not applicable.

Acknowledgments: We would like to thank the reviewers for their careful reading of the manuscript and their suggestions which have improved the presentation of this work.

Conflicts of Interest: The authors declare no conflict of interest.

\section{References}

1. Wiener, H. Structural determination of paraffin boiling points. J. Am. Chem. Soc. 1947, 69, 17-20. [CrossRef]

2. Devillers, J.; Balaban, A.T. (Eds.) Topological Indices and Related Descriptors in QSAR and QSPR; Gordon and Breach: Amsterdam, The Netherlands, 1999.

3. Karelson, M. Molecular Descriptors in QSAR/QSPR; Wiley-Interscience: New York, NY, USA, 2000.

4. Todeschini, R.; Consonni, V. Handbook of Molecular Descriptors; Wiley-VCH: Weinheim, Germany, 2000.

5. Gutman, I.; Furtula, B.; Katanić, V. Randić index and information. AKCE Int. J. Graphs Comb. 2018, 15, 307-312. [CrossRef]

6. Estrada, E. Quantifying network heterogeneity. Phys Rev. E 2010, 82, 066102. [CrossRef] 
7. Pineda, J.; Martínez, C.; Mendez, A.; Muños, J.; Sigarreta J.M. Application of Bipartite Networks to the Study of Water Quality. Sustainability 2020, 12, 5143. [CrossRef]

8. Li, X.; Zhao, H. Trees with the first smallest and largest generalized topological indices. MATCH Commun. Math. Comput. Chem. 2004, 50, 57-62.

9. Miličević, A.; Nikolić, S. On variable Zagreb indices. Croat. Chem. Acta 2004, 77, 97-101.

10. Zhou, B.; Trinajstić, N. On general sum-connectivity index. J. Math. Chem. 2010, 47, 210-218. [CrossRef]

11. Vukičević, D.; Gašperov, M. Bond Additive Modeling 1. Adriatic Indices. Croat. Chem. Acta 2010, 83, $243-260$.

12. Vukičević, D. Bond additive modeling 2. Mathematical properties of max-min rodeg index. Croat. Chem. Acta 2010, 83, 261-273.

13. Furtula, B.; Das, K.C.; Gutman, I. Comparative analysis of symmetric division deg index as potentially useful molecular descriptor. Int. J. Quantum Chem. 2018, 118, e25659. [CrossRef]

14. Vukičević, D.; Furtula, B. Topological index based on the ratios of geometrical and arithmetical means of end-vertex degrees of edges. J. Math. Chem. 2009, 46, 1369-1376. [CrossRef]

15. Das, K.C. On geometric-arithmetic index of graphs. MATCH Commun. Math. Comput. Chem. 2010, 64, 619-630. [CrossRef]

16. Das, K.C.; Gutman, I.; Furtula, B. Survey on Geometric-Arithmetic Indices of Graphs. MATCH Commun. Math. Comput. Chem. 2011, 65, 595-644.

17. Das, K.C.; Gutman, I.; Furtula, B. On first geometric-arithmetic index of graphs. Discrete Appl. Math. 2011, 159, 2030-2037. [CrossRef]

18. Martínez-Pérez, A.; Rodríguez, J.M.; Sigarreta, J.M. A new approximation to the geometric-arithmetic index. J. Math. Chem. 2018, 56, 1865-1883. [CrossRef]

19. Mogharrab, M.; Fath-Tabar, G.H. Some bounds on GA index of graphs. MATCH Commun. Math. Comput. Chem. 2010, 65, 33-38.

20. Rodríguez, J.M.; Sigarreta, J.M. Spectral properties of geometric-arithmetic index. Appl. Math. Comput. 2016, 277, 142-153. [CrossRef]

21. Sigarreta, J.M. Bounds for the geometric-arithmetic index of a graph. Miskolc Math. Notes 2015, 16, 1199-1212. [CrossRef]

22. Shegehall, V.S.; Kanabur, R. Arithmetic-geometric indices of path graph. J. Math. Comput. Sci. 2015, 16, 19-24.

23. Shegehall, V.S.; Kanabur, R. Arithmetic-geometric indices of graphs with pendant vertices attached to the middle vertices of path. J. Math. Comput. Sci. 2015, 6, 67-72.

24. Shegehall, V.S.; Kanabur, R. Computation of new degree-based topological indices of graphene. J. Math. 2016, $2016,4341919$. [CrossRef]

25. Zheng, L.; Tian, G.-X.; Cui, S.-Y. On spectral radius and energy of arithmetic-geometric matrix of graphs. MACTH Commun. Math Comput. Chem. 2020, 83, 635-650.

26. Guo, X.; Gao, Y. Arithmetic-geometric spectral radius and energy of graphs. MACTH Commun. Math. Comput. Chem. 2020, 83, 651-660.

27. Das, K.C.; Gutman, I. Degree-based energies of graphs. Linear Algebra Appl. 2018, 554, 185-204. [CrossRef]

28. Vujošević, S.; Popivoda, G.; Vukićević, K.; Furtula, B.; Škrekovski, R. Arithmetic-geometric index and its relations with geometricarithmetic index. Appl. Math. Comput. 2021, 391, 125706. [CrossRef]

29. Carballosa, W.; Granados, A.; Méndez-Bermúdez, J.A.; Pestana, D.; Portilla, A. Computational properties of the arithmeticgeometric index. Submitted.

30. Cui, S.-Y.; Wang, W.; Tian, G.-X.; Wu, B. On the arithmetic-geometric index of graphs. MATCH Commun. Math. Comput. Chem. 2021, 85, 87-107.

31. Milovanović, I.; Matejić, M.; Milovanović, E.I. Upper bounds for arithmetic-geometric index of graphs. Sci. Publ. State Univ. Novi Pazar Ser. A Appl. Math. Inf. Mech. 2018, 10, 49-54. [CrossRef]

32. Rodríguez, J.M.; Sánchez, J.L.; Sigarreta, J.M.; Tourís, E. Bounds on the arithmetic-geometric index. Symmetry 2021, 13, 689. [CrossRef]

33. Poojary, P.; Raghavendra, A.; Shenoy, B.G.; Farahani, M.R.; Sooryanarayana, B. Certain topological indices and polynomials for the Isaac graphs. J. Discr. Math. Sci. Crypt. 2021, 24, 511-525.

34. I. Gutman, Relation between geometric-arithmetic and arithmetic-geometric indices. J. Math. Chem. 2021. [CrossRef]

35. Milovanović, I.; Milovanović, E.I.; Matejić, M.M. On Upper Bounds for the Geometric-Arithmetic Topological Index. MATCH Commun. Math. Comput. Chem. 2018, 80, 109-127. 